\title{
Ecología trófica del Liso (Rhamdia quelen Quoy \& Gaimard, 1824) en el río Sinú, Colombia
}

\author{
Trophic ecology of Liso (Rhamdia quelen Quoy \& Gaimard, 1824) \\ in the Sinu river, Colombia
}

\author{
Charles W. Olaya-Nieto, ${ }^{1 *}$ M.Sc, Liliana Pacheco-Orozco, ${ }^{1}$ Prof en Acuicultura, \\ Julieth Ochoa-Arteaga, ${ }^{1}$ Prof en Acuicultura.
}

\begin{abstract}
${ }^{1}$ Universidad de Córdoba, Departamento de Ciencias Acuícolas, Laboratorio de Investigación Biológico Pesquera-LIBP. Lorica, Colombia.*Correspondencia: charles_olaya@hotmail.com
\end{abstract}

Recibido: Diciembre de 2011; Aceptado: Agosto de 2012.

\section{RESUMEN}

Objetivo. Estudiar la ecología trófica del Liso en el río Sinú como contribución al conocimiento de su biología y ecología, a su preservación en el medio natural y al ordenamiento de su pesquería en la cuenca. Materiales y métodos. El contenido estomacal se evaluó con el Coeficiente de vacuidad, Grado de digestión, Frecuencia de ocurrencia, Frecuencia numérica, Gravimetría, Índice de importancia relativa (IIR) y la relación longitud intestinal (LI)-longitud total (LT). Resultados. El $38.2 \%$ de los estómagos estaban vacíos y el $92.5 \%$ de las presas estaban medio digeridas, identificándose 5 grupos alimentarios: Peces, Crustáceos, Insectos, Material vegetal y Otros. Peces, conformado por Cachana, Cocobolo, Mayupa, Sardina y Restos de peces, fue la presa más frecuente $(69.9 \%)$, abundante (45.5\%) y con mayor composición por peso $(76.8 \%)$. El Índice de importancia relativa (IIR) indica que Peces fue el alimento principal (IIR $=53.7 \%$ ), y que las demás presas eran de baja importancia relativa, circunstanciales o incidentales. Conclusiones. Los resultados alcanzados en este estudio indican que el Liso es un pez con hábitos alimentarios nocturnos y carnívoros, con tendencia a consumir Peces.

Palabras clave: Rhamdia quelen, Alimentación, Colombia (Fuente:AIMS).

\section{ABSTRACT}

Objective. To study the trophic ecology of Liso in the Sinu river as a contribution to the knowledge of its biology and ecology, preservation in wild environment and the management of fishery in the basin. Materials and methods. The stomach content was analyzed by using the Proportion of empty stomachs, digestion rate, Frequency of occurrence, numerical Frequency, Gravimetry, relative importance Index (RII) and gut length (GL)-total length (TL) relationship. Results. $38.2 \%$ of stomachs were empty, $92.5 \%$ of preys were half digested and five food items were identified, they are conformed by Fish, Crustaceans, Insects, Vegetable Rests and Others. Fish, conformed by Cachama, Cocobolo, Mayupa, Sardine and Rest of other fish, was the most common prey (69.9\%), the most abundant prey $(45.5 \%)$ and the greatest prey composition in weight (76.8\%). Relative importance Index indicated that fish was the main prey (RII $=81.6 \%$ ), and the other prey reached low relative importance, or circumstantial or incidental prey. Conclusions. The results reached in this study indicate that the Liso is a fish with nocturnal and carnivorous feeding habits, with a tendency to consume other fish.

Key words: Rhamdia quelen, Feeding, Colombia (Source:AIMS). 


\section{INTRODUCCIÓN}

El Liso (Rhamdia quelen Quoy \& Gaimard, $1824)$ es una especie común en las corrientes pequeñas en el río Sinú, de carne muy fina y generalmente apetecida, pero su relativamente baja abundancia ha impedido que tenga una mayor importancia comercial (1). Sin embargo, se le ha reconocido algún valor dentro de la pesca doméstica o de subsistencia (2); además de que puede ser utilizada en la piscicultura de ornamentación.

Pertenece a la familia Heptapteridae (3), alcanza $35.0 \mathrm{~cm}$ de longitud estándar (1), presenta una cabeza aplanada dorso-ventralmente, vientre de color claro, sin banda lateral diferenciada, aleta caudal bifurcada y lóbulos terminados en punta. Los barbicelos maxilares alcanzan o sobrepasan la aleta caudal y el quinto radio del lóbulo superior de la aleta caudal es el más largo (4).

Se distribuye en las cuencas del Atrato, Cauca, Magdalena, San Jorge, Sinú y Catatumbo, Orinoco y Amazonas $(1,2,4-7)$, con marcada preferencia por los ambientes de corrientes rápidas y someras de piedemonte, o de baja corriente de pequeñas y medianas quebradas 0 grandes ríos (8).

La mayoría de las especies del género Rhamdia son de ciclo reproductivo corto, con la primera maduración gonadal al final del primer año de vida, coincidiendo con la estación lluviosa (9). El Liso es un pez con tipo de desarrollo ovocitario asincrónico en más de dos grupos, con desoves parciales durante todo el año, con talla media de madurez sexual de $24.0 \mathrm{~cm}$ de longitud total, ovocitos maduros con $963 \mu$ y fecundidad promedio estimada en 26.305 ovocitos (10).

El objetivo de este trabajo fue estudiar la ecología trófica del Liso en el Bajo río Sinú, como contribución al conocimiento de su biología y ecología, a su preservación en el medio natural, al ordenamiento de su pesquería y a su aprovechamiento pesquero y acuícola en la cuenca.

\section{MATERIALES Y MÉTODOS}

Localización y descripción del área de estudio. El estudio se desarrolló en el Bajo río Sinú, entre enero y diciembre 2005. El río Sinú tiene una longitud de $380 \mathrm{Km}$, extendiéndose desde el nudo de Paramillo en el departamento de Antioquia hasta el delta de Tinajones en el mar Caribe, en el departamento de Córdoba, cuya cuenca tiene una superficie de $13700 \mathrm{~km}^{2}$. En su parte media y baja se encuentran la ciénaga
Grande de Betancí, la ciénaga Grande de Lorica, las ciénagas de la margen izquierda y otras de menor tamaño. Presenta una temperatura promedio anual de $28^{\circ} \mathrm{C}$, que en épocas de lluvias cuando las aguas inundan los planos cenagosos, disminuye a $27^{\circ} \mathrm{C}$ (11). La pluviosidad media anual en las zonas en las zonas bajas es de

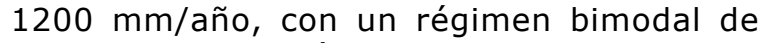
precipitaciones, períodos lluviosos en abril-junio y agosto-octubre. El principal período seco se prolonga desde noviembre a marzo, con uno de menor proporción en julio-agosto (12).

Obtención de las muestras. Para la identificación taxonómica de la especie se siguió la clasificación de Bockmann \& Guazzelli (3), colectándose 338 individuos entre enero y diciembre 2005, a quienes se les tomó longitud total (LT), longitud horquilla (LH) y longitud estándar (LS) al milímetro más cercano con un ictiómetro graduado en $\mathrm{mm}$ (Tridente, España), y el peso total (WT) al gramo más cercano con una balanza eléctrica (Ohaus, USA) con capacidad de $1500 \mathrm{~g}( \pm 1 \mathrm{~g})$.

Extracción de los estómagos. Aplicando las técnicas de Laevastu (13), una vez efectuada la disección de los peces, se ubicaron las diferentes partes del tubo digestivo. Luego se procedió a retirar el estómago y el intestino para conservarlos en frascos plásticos de $250 \mathrm{ml}$ que contenían formol al $10 \%$ bufferado. Todos los frascos fueron rotulados, indicando la especie, número de la muestra y fecha. En un formato se anotó la información citada arriba más el sitio de captura, arte de pesca utilizado, talla, peso total y peso eviscerado.

Análisis del contenido estomacal. El contenido estomacal se colocó en una caja Petri y se examinó al estereoscopio (Leica, USA) y microscopio (Leica, USA), separando, identificando y enumerando el alimento o presas presentes. La identificación se efectuó hasta el nivel taxonómico permitido por el grado de digestión del alimento, ordenándose en grupos, ítems o presas, pesándose en una balanza eléctrica (Ohaus, USA) de 1500 $( \pm 0.01 \mathrm{~g})$ de capacidad. El material animal que estaba totalmente digerido se identificó por los fragmentos, en lo posible (14).

El Coeficiente de vacuidad (CV) se obtuvo con la técnica de Windell (15):

$\mathrm{CV}=100 *$ No. estómagos vacíos / No. total de estómagos analizados

El Grado de digestión (GD) se evaluó con la escala de Laevastu (13), la cual clasifica el estado de las presas así: Fresco, Medio digerido y Digerido. 
Se utilizaron tres métodos para cuantificar el contenido estomacal, expresado en valores promedios mensuales y anuales: Frecuencia de ocurrencia (FO), Frecuencia numérica (FN) y Gravimetría (G) (15):

FO $=100 *$ Ocurrencia de presas del ítem A/ Número total de estómagos con alimento

FN $=100 *$ Número de presas del ítem A/Número total de presas

$\mathrm{G}=100 *$ Peso de las presas del ítem A/Peso de todas las presas

Para establecer la importancia de cada presa en la composición de la dieta se estimó el Índice de importancia relativa (IIR) (16) modificado (17): IIR $=F^{*} G / 100$, en donde, IIR representa el Indice de importancia relativa, $F$ es el porcentaje de la Frecuencia de ocurrencia y $G$ es el porcentaje de la Gravimetría. Esta expresión presenta un rango de 0 a 100 , en donde el rango de 0 a $10 \%$ representa grupos tróficos de importancia relativa baja, de 10 a $40 \%$ grupos de importancia relativa secundaria y 40 a $100 \%$ grupos de importancia relativa alta.

Se estableció la relación longitud intestinallongitud total de acuerdo con la escala de Brusle (18) y se analizaron las preferencias alimentarias de acuerdo con las tallas que presenta la especie en estudio y con el ciclo hidrológico del río Sinú. Se utilizó estadística descriptiva, expresando las variables como media \pm desviación estándar y se estimó el coeficiente de correlación ( $r$ ) a la relación longitud intestinal-longitud total.

\section{RESULTADOS}

Se analizaron 338 estómagos de individuos capturados en el período comprendido entre enero y diciembre 2005. La talla mínima registrada fue de $15.5 \mathrm{~cm}$ LT (abril), la máxima de $37.0 \mathrm{~cm} \mathrm{LT} \mathrm{(abril);} \mathrm{mientras} \mathrm{que} \mathrm{el} \mathrm{menor} \mathrm{peso}$ fue de $28.0 \mathrm{~g}$ (abril) y el mayor de $486.0 \mathrm{~g}$ (junio). La distribución de frecuencias de tallas mostró rangos entre 15.0 y $35.0 \mathrm{~cm} \mathrm{LT}$, con talla media de captura de $23.7 \mathrm{~cm}$ LT (Figura 1); mientras que la distribución de frecuencias de pesos presenta rangos entre 50.0 y $500.0 \mathrm{~g}$ y peso promedio de $139.2 \mathrm{~g}$.

Del total de estómagos estudiados, el $38.2 \%$ se encontró vacío, destacándose abril (54.2\%), marzo $(53.8 \%)$ y junio $(53.3 \%)$. El $92.5 \%$ del alimento consumido se encontró medio digerido, alcanzando su valor máximo mensual en diciembre (100\%), el $5.9 \%$ fresco, y solo el $1.6 \%$ digerido.

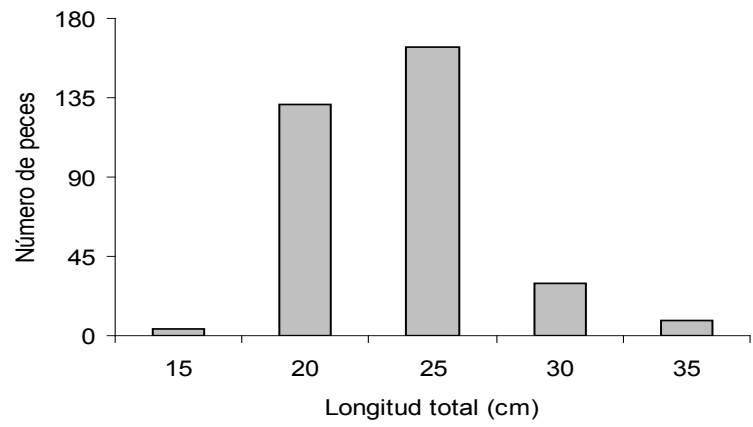

Figura 1. Distribución de frecuencia de tallas del Liso en el río Sinú. Año 2005.

Fueron identificados cinco grupos alimentarios: Peces, Crustáceos, Material vegetal, Insectos y Otros, siendo Peces el más frecuente (69.9\%) (Figura 2) con individuos pertenecientes a cuatro géneros: Cachana (Cynopotamus atratoensis), Cocobolo (Andinoacara pulcher), Mayupa (Sternopygus macrurus), Sardina (Astyanax sp) y Restos de peces; seguido por Material vegetal $(36.4 \%)$, conformado por Semillas y Restos vegetales; Crustáceos (18.2\%), constituido por Camarón y Cangrejo; Insectos (16.7\%) conformado por Diptera, Coleoptera, Hemiptera, Odonata y Restos de insectos y Otros (12.0\%), constituido por Detritos, Gusanos, Moluscos, Piel de serpiente, Restos de huesos, Restos de vísceras y Sedimento. Peces y Material vegetal estuvieron presentes durante todos los meses del año de estudio, mientras que Crustáceos estuvo ausente en marzo y abril, Insectos en marzo y agosto, y Otros en enero y abril.

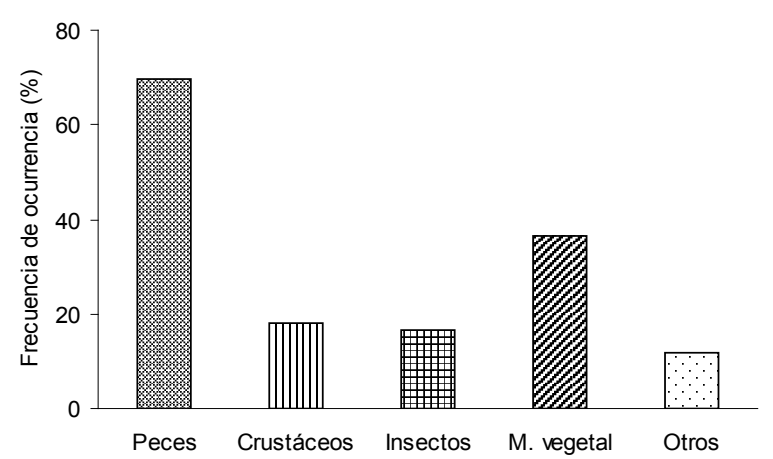

Figura 2. Frecuencia de ocurrencia de presas en el estómago del Liso en el río Sinú.

Peces $(45.5 \%)$ fue el grupo más abundante seguido por Material vegetal (24.0\%), Crustáceos $(11.8 \%)$, Insectos $(10.9 \%)$ y Otros $(7.8 \%)$ (Figura 3). En la composición mensual se observó que Material vegetal y Crustáceos fueron las presas más abundantes en aguas ascendentes y aguas altas. Peces fue también el grupo con mayor composición en peso $(76.8 \%)$, seguido 


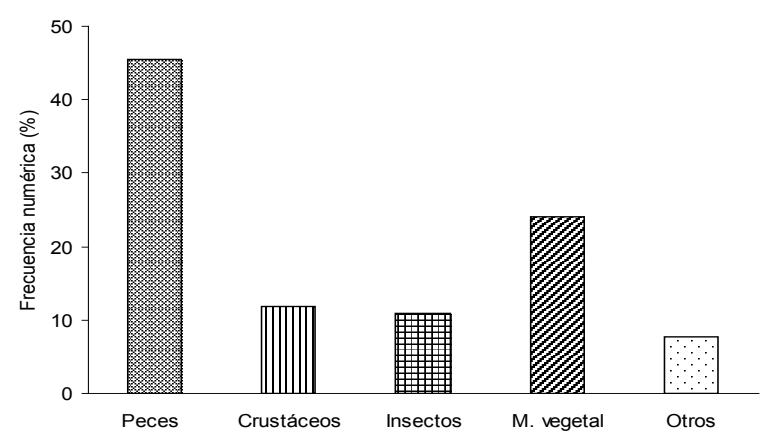

Figura 3. Frecuencia numérica de presas en el estómago del Liso en el río Sinú.

por Crustáceos (9.8\%), Otros (6.7\%), Material vegetal $(3.7 \%)$ e Insectos $(3.1 \%)$, mostrando los mayores valores en todos los meses (Figura 4).

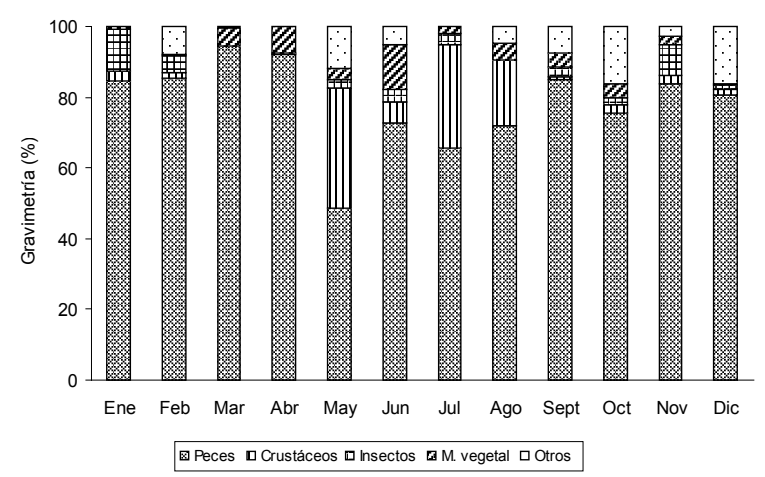

Figura 4. Gravimetría de presas en el estómago del Liso en el río Sinú.

El Índice de importancia relativa (IIR) indicó que Peces fue el alimento principal del Liso (IIR $=53.7 \%$ ). Crustáceos (IIR $=1.8 \%$ ), Material vegetal (IIR $=1.3 \%)$, Otros (IIR $=0.8 \%$ ) e Insectos (IIR $=0.5 \%$ ) fueron presas de baja importancia relativa, circunstanciales o incidentales. La relación longitud intestinallongitud total (LI-LT) fue estimada en 1.0, lo que clasifica a la especie en estudio como carnívora de acuerdo con la escala de Brusle (18).
Para analizar las preferencias alimentarias con respecto a la talla, los ejemplares de la especie en estudio se agruparon en cinco intervalos de tallas, los cuales oscilaron entre 12.5 y 37.5 $\mathrm{cm}$ LT, en donde solo se observó el consumo de Peces (100\%) para el intervalo 12.5-17.5 $\mathrm{cm}$, mientras que en los siguientes (17.5-22.5, 22.5-27.5 y 27.5-32.5 cm) consumieron los cinco grupos alimentarios (Peces, Crustáceos, Insectos, Material vegetal y Otros), y en el intervalo mayor $(32.5-37.5 \mathrm{~cm})$ consumieron Peces (50.0\%), Material vegetal (30.0\%), Crustáceos y Otros (10.0\%).

En la tabla 1 se muestra la composición y tamaño de los peces consumidos por el Liso. Entre 12.517.5 y $27.5-32.5 \mathrm{~cm}$ no se registraron peces enteros, sino restos de ellos; entre $17.5-22.5 \mathrm{~cm}$ se encontró Mayupa (6.0\%), Sardina (1.5\%) y Restos de peces (92.5\%); entre $(22.5-27.5 \mathrm{~cm})$ se encontró Cachana (1.6\%), Cocobolo (3.1\%), Mayupa $(4.7 \%)$, Sardina (1.6\%) y Restos de peces $(89.1 \%) ; y$ entre $(32.5-37.5 \mathrm{~cm})$ se observó únicamente Cocobolo $(20.0 \%)$ y Restos de peces $(80.0 \%)$.

La talla del Liso osciló entre 15.5 y $37.0 \mathrm{~cm} \mathrm{LT}$, mientras que la talla de los diferentes peces consumidos se encontró entre 2.5 y $12.0 \mathrm{~cm}$ $\mathrm{LT}$, observándose que a medida que aumentó la talla del predador disminuyó la talla de la presa consumida; lo que se confirmó con la relación talla media de la presa/talla media del predador, la cual muestra correlación inversa.

De acuerdo con los niveles durante el ciclo hidrológico del río: aguas bajas (diciembre, enero, febrero), aguas ascendentes (marzo, abril, mayo), aguas altas (junio, julio, agosto) y aguas descendentes (septiembre, octubre, noviembre), se observó que Peces y Crustáceos fueron los grupos más consumidos en aguas altas; mientras que Material vegetal lo fue en aguas ascendentes y aguas altas; Insectos, en aguas bajas; Otros, en aguas descendentes (Figura 5).

Tabla 1. Composición y tamaño de los peces consumidos por el Liso en el río Sinú.

\begin{tabular}{|c|c|c|c|c|c|c|c|c|c|}
\hline \multirow{2}{*}{$\begin{array}{c}\text { Liso } \\
\text { LT (cm) }\end{array}$} & \multirow[b]{2}{*}{$\mathbf{n}$} & \multicolumn{4}{|c|}{ Presas consumidas } & \multicolumn{4}{|c|}{ Tamaño presa (LT) } \\
\hline & & $\begin{array}{c}\text { Cachana } \\
(\%)\end{array}$ & $\begin{array}{c}\text { Cocobolo } \\
(\%)\end{array}$ & $\begin{array}{c}\text { Mayupa } \\
(\%)\end{array}$ & $\begin{array}{c}\text { Sardina } \\
(\%)\end{array}$ & $\begin{array}{c}\text { R. peces } \\
(\%)\end{array}$ & $\begin{array}{l}\text { Mín. } \\
\text { (cm) }\end{array}$ & $\begin{array}{l}\text { Máx. } \\
(\mathrm{cm})\end{array}$ & $\begin{array}{l}\text { Prom. } \\
(\mathrm{cm})\end{array}$ \\
\hline $12.5-17.5$ & 3 & - & - & - & - & 100.0 & - & - & - \\
\hline $17.5-22.5$ & 67 & - & - & 6.0 & 1.5 & 92.5 & 2.5 & 12.0 & 7.3 \\
\hline $22.5-27.5$ & 64 & 1.6 & 3.1 & 4.7 & 1.6 & 889.1 & 3.0 & 10.5 & 6.8 \\
\hline $27.5-32.5$ & 7 & - & - & - & - & 100.0 & - & - & - \\
\hline $32.5-37.5$ & 5 & - & 20.0 & - & - & 80.0 & 6.5 & 6.5 & 6.5 \\
\hline
\end{tabular}




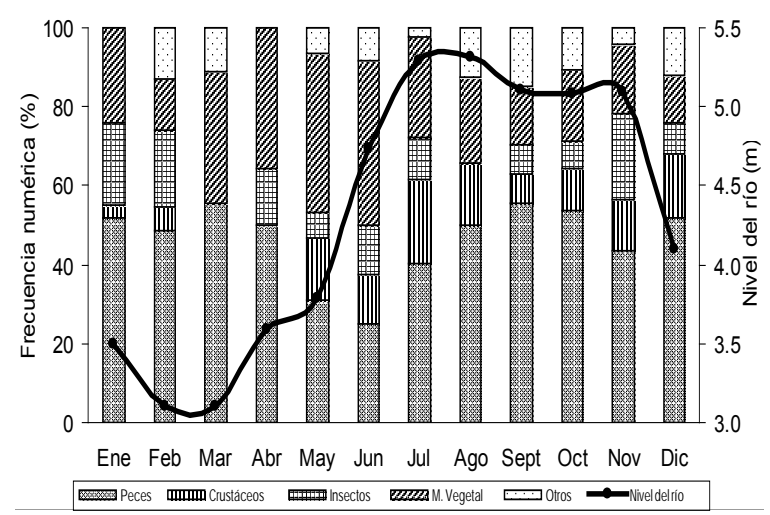

Figura 5. Preferencias alimentarias del Liso y el ciclo hidrológico del río Sinú.

\section{DISCUSIón}

El Coeficiente de vacuidad encontrado en este trabajo es mayor que los reportados para la especie en los ríos San Martín y Guarayos $(16.7 \%)$ en Bolivia (19), en el río Sauce Grande (0\%) en Argentina (20). La presencia de altos porcentajes de estómagos vacíos puede relacionarse con la reducción de la actividad alimentaria como consecuencia de la actividad reproductiva, lo que sugiere que los coeficientes de vacuidad pueden estar asociados con los procesos reproductivos de la especie (21). De igual manera, altos porcentajes de vacuidad se podrían presentar debido a que los peces carnívoros tienen un estómago evolucionado que segrega ácidos para digerir rápidamente hueso, carne y escamas de las presas ingeridas, y posee un intestino mucho más corto que las especies herbívoras, por lo que la digestión es más rápida (22).

El alto porcentaje de presas digeridas y medio digeridas, al igual que el alto Coeficiente de vacuidad, pueden estar relacionados con el intestino corto que presentan los peces depredadores, mientras que su estómago es más voluminoso o evolucionado con $\mathrm{pH}$ ácido y presencia de jugos gástricos, favoreciendo una digestión muy rápida (22). También, la boca, la cavidad bucal y la faringe están asociadas con la secreción, captura, orientación y preparación predigestiva del alimento (23).

Diferentes trabajos realizados en América del Sur coinciden con los resultados obtenidos en este estudio puesto que los grupos alimentarios son muy similares. Varios autores han reportado que Rhamdia quelen se alimenta de Insectos acuáticos, Peces y Material vegetal como frutos, semillas y flores en el río Cauca, Colombia, mostrando una dieta muy flexible (24) y de Algas, Detritos orgánicos, Restos inorgánicos y Restos vegetales en Bolivia (19).
En investigaciones realizadas en Brasil se ha observado que los adultos son omnívoros, con una tendencia al carnivorismo, con clara preferencia por Peces, Crustáceos, Moluscos, Insectos terrestres y acuáticos, Restos vegetales y Detritus orgánicos (25-26). Otros autores reportaron que Rhamdia quelen es una especie piscívora en el Alto río Paraná (27); que presenta una dieta diversificada compuesta, tanto por grupos de origen animal (Camarón e Insectos), como vegetal (Macrofitas), ubicándolo como un pez carnívoro generalista (28); que los organismos encontrados en su contenido gastrointestinal no están restringidos a hábitats bentónicos, lo que indica que esta especie es generalista en lo que respecta a la elección de sus alimentos (25), observándosele hábitos alimentarios nocturnos en este estudio, lo cual coincide con lo reportado $(19,24)$.

El Índice de importancia relativa estimado en este estudio (IIR Peces $=53.7 \%$ ) es muy diferente al reportado por López et al. (20), quienes afirman que el alimento dominante o principal de la especie en el río Sauce Grande (Argentina) es Moluscos (Chilina parchappii) (56.4\%) mientras que Ephemeroptera (Baetis inops), Odonata (Aeshna bonariensis) y Trichoptera (Neotrichia $\mathrm{sp)}$ fueron alimentos ocasionales.

La relación longitud intestinal-longitud total estimada (LI-LT $=1.0$ ), que clasifica al Liso como un pez carnívoro, es mayor que la relación encontrada para la especie en los ríos Paraibuna $($ LI-LT $=0.83)$ y Grande $($ LI-LT $=0.75)(26)$, correspondientes a especies omnívoras con tendencia de ingestión de presas de origen animal (Figura 6).

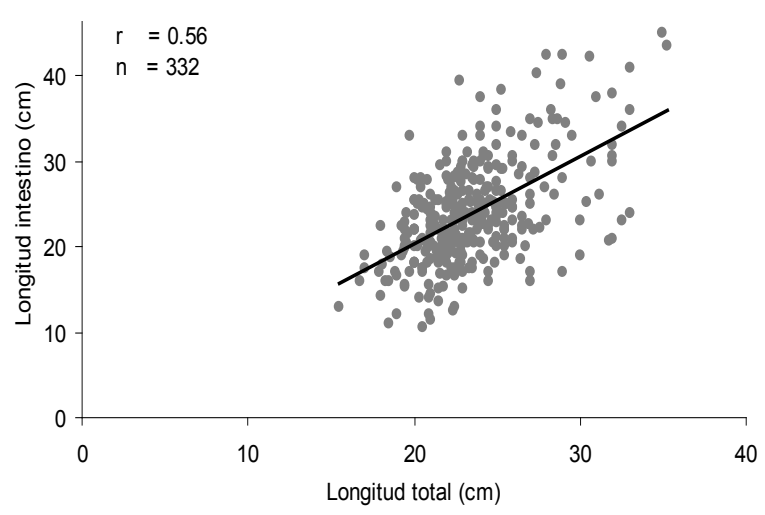

Figura 6. Relación longitud intestino-longitud total del Liso en el río Sinú. 
Los peces carnívoros presentan un intestino corto debido a que la cantidad de alimento es menor y la calidad superior, lo que hace que el tránsito sea más lento, aspecto importante para favorecer la difusión de los nutrientes (29); de igual forma, la longitud del intestino parece estar más relacionada con la cantidad de material indigerible del alimento, que con su origen animal y/o vegetal. Para el Liso se encontró correlación positiva $(r=0.56)$ entre el tamaño del intestino y su talla.

La talla del Liso osciló entre 15.5 y $37.0 \mathrm{~cm} \mathrm{LT}$, mientras que la talla de los diferentes peces consumidos se encontró entre 2.5 y $12.0 \mathrm{~cm}$ LT, observándose que a medida que aumenta la talla del predador disminuye la talla de la presa consumida; lo que se confirmó con la relación talla media de la presa/talla media del predador, la cual muestra correlación inversa. La morfología del pez juega un papel importante en el tipo de presa consumida, en tanto que las variaciones morfológicas pueden conducir a cambios en su capacidad de alimentación y a la subsecuente explotación diferencial de los recursos alimentarios (30). El comportamiento alimentario es característico de cada especie. A medida que se hacen más estables sus condiciones de alimentación, se reduce la diversidad de alimentos consumidos, por lo que a mayor variabilidad de alimento disponible es mayor la diversidad de elementos alimentarios ingeridos (23).

Los resultados obtenidos indican que Peces es la presa más consumida, lo que sumado -a la información arrojada por el Índice de importancia relativa y la relación longitud intestino-longitud total, permite inferir que el Liso es un pez con hábitos alimentarios nocturnos y carnívoros con tendencia a consumir Peces.

\section{Agradecimientos}

Los autores expresan sus agradecimientos a los pescadores y comercializadores de pescado de la cuenca baja del río Sinú y a los tesistasinvestigadores involucrados en el proyecto de investigación "Estimación de los parámetros biológicos básicos de peces comerciales del Río Sinú-Fase II", Código FMV-01-04, Numeral 1120107, del cual hace parte este trabajo. A la Universidad de Córdoba, por la financiación recibida.

\section{REFERENCIAS}

1. Dahl G. Los peces del norte de Colombia. Bogotá: Inderena; 1971.

2. Dahl G, Medem F. Informe sobre la fauna acuática del Río Sinú. Bogotá: Departamento de Investigaciones Ictiológicas y Faunísticas. Corporación Autónoma Regional de los Valles del Magdalena y del Sinú-CVM; 1964.

3. Bockmann FA, Guazzelli GM. Family Heptapteridae (Heptaterids). In: Reis RE, Kullander SO, Ferraris $\mathrm{CJ} J$ r. (eds.). Checklist of the freshwater fishes of South and Central America. Porto Alegre: Editora da Pontifícia Universidade Católica do Rio Grande do Sul-EDIPUCRS; 2003: 406-431.

4. Galvis G, Mojica JI, Camargo M. Peces del Catatumbo. Bogotá: Asociación Cravo Norte; 1997.

5. Miles C. Peces del Río Magdalena. Bogotá: Ministerio de Economía Nacional, Sección de Piscicultura, Pesca y Caza; 1947.
6. Silfvergrip AMC. A systematic revision of the Neotropical catfish genus Rhamdia (Teleostei, Pimelodidae). Stockholm: Swedish Museum of Natural History; 1996.

7. Lehmann P. Composición y estructura de las comunidades de peces de los tributarios en la parte alta del río Cauca, Colombia. [Trabajo de pregrado]. Cali: Universidad del Valle, Facultad de Ciencias; 1999.

8. Ortega-Lara A. Murillo O, Pimienta C, Sterling E. Los peces del alto Cauca. Catálogo de especies. Cali: Informe presentado a la Corporación Autónoma Regional del Valle del Cauca, CVC; 1999.

9. Gomes LC, Gomes ARC, Golombieski JI, Baldisserotto B. Rhamdia quelen (Pimelodidae), espécie promissora para a piscicultura do sul do Brasil-uma revisão. In: XIII Encontro Brasileiro de Ictiologia. São Carlos. 1999; 535 (Resumo). 
10. Olaya-Nieto CW, Hernández DF, Ayarza E. Biología reproductiva del Liso Rhamdia quelen (Pisces: Heptapteridae) en el río Sinú, Colombia. Acta biol Colomb 2010: 15(3):61-74.

11. Bustamante ID. Los suelos de la cuenca del Río Sinú y el Proyecto Urrá I. Temas Agrarios 2000; 9:15-28.

12. IDEAM. Base de datos. Barranquilla; 1998.

13. Laevastu T. Manual de métodos de biología pesquera. Zaragoza: Editorial Acribia; 1980.

14. Lugo RL. Determinación de hábitos, madurez sexual y desove en tres especies ícticas de la cuenca del Río Tomo (Vichada) y consideraciones para el mantenimiento de los padrotes. Villavicencio: Universidad Tecnológica de los Llanos Orientales-I.I.O.C. N²; 1989.

15. Windell JT. Food analysis and rate of digestion. In: Ricker WE. (ed.). Methods for assessment of fish production in fresh waters. 2nd edition. Oxford: Blackwell Scientific Publications; 1971.

16. Yáñez-Arancibia A, Curiel-Gómez J, Leyton V. Prospección biológica y ecología del bagre marino Galeichthys caerulescens (Günther) en el sistema lagunar costero de Guerrero, México (Pisces: Ariidae). An Centro Cienc del Mar y Limnol Univ Nal Autón México 1976; 3(1):125-180.

17. Olaya-Nieto CW, Tobías-Arias AJ, SeguraGuevara F, Brú-Cordero SB, Tordecilla-Petro G. Modificación del índice de importancia relativa (IIR) de Yáñez-Arancibia, Curiel-Gómez \& Leyton (1976). Lorica: Laboratorio de Investigación Biológico Pesquera-LIBP. Departamento de Acuicultura, Facultad de Medicina Veterinaria y Zootecnia, Universidad de Córdoba; 2003.

18. Brusle J. Food and feeding in grey mullet. In: Oren $\mathrm{OH}$. (ed.). Aquaculture of Grey Mullet. Cambridge: Cambridge University Press; 1981.

19. Rebolledo PP. Levantamiento de base de la ictiofauna en los ríos San Martín y Guarayos en la Concesión Oquiriquia. Proyecto BOLFOR. USAID-Gobierno de Bolivia. Documento Técnico 1995; (22):1-55.

20. López A, Durán W, Tejera L. Alimentación de la ictiofauna del río Sauce Grande, provincia de Buenos Aires, Argentina. Biología Acuática 2003; 20: 73-79.
21. Hynes HBN. The food of fresh-water sticklebacks (Gasterosteus aculeatus and Pygosteus pungitius), with a review of methods used in studies of the food of fishes. J Anim Ecol 1950; 19(1):36-58.

22. Lagler KF, Bardach JE, Miller RR, May-Passino DR. Ictiología. Mexico: AGT; 1984.

23. Prejs A, Colomine G. Métodos para el estudio de los alimentos y las relaciones tróficas de los peces. Caracas: Universidad Central de Venezuela; 1981.

24. Ortega-Lara A. Aguiño A, Sánchez GC. Caracterización de la ictiofauna nativa de los principales ríos de la cuenca alta del río Cauca en el departamento del Cauca. Informe presentado a la Corporación Autónoma Regional del Cauca, CRC. Popayán: Fundación para la Investigación y el Desarrollo Sostenible-Funindes; 2002.

25. García DJ. Aspectos biológicos del Bagre negro con énfasis en su alimentación. [Tesis de Licenciatura]. Montevideo: Universidad de la República-UDELAR, Facultad de Ciencias; 1995.

26. Gomiero LM, Souza UP, Braga FMS. Reprodução e alimentação de Rhamdia quelen (Quoy \& Gaimard, 1824) em rios do Núcleo Santa Virgínia, Parque Estadual da Serra do Mar, São Paulo, SP. Biota Neotrop 2007; 7(3):127-133.

27. Hahn NS, Fugi R, Peretti D, Russo MR, Loureiro-Crippa VE. Estrutura trófica da ictiofauna da planicie de inundação do alto Rio Paraná. In: Agostinho AA, Thomaz SM, Rodrigues L, Gomes LC. (eds.). A Planicie de Inundação do Alto rio Paraná. Maringá: Area de Pesquisas Ecológicas de Longa Duração, Núcleo de Pesquisas em Limnologia, Ictiologia e Aqüicultura-Nupelia, Universidade Estadual de Maringá; 2002: 123-126.

28. Pereira CCGF, Smith WS, Espíndola ELG. Hábitos alimenticios de nueve especies del embalse de Três Irmãos, São Paulo, Brasil. Universidad y Ciencia [Número Especial I]; 2004: 33-38.

29. Rotta MA. Aspectos gerais da fisiología e estrutura do sistema digestivo dos peixes relacionados à piscicultura. Corumbá: Embrapa Pantanal Documentos; 2003(53): 1-48.

30. Magnhagen C, Heibo E. Gape size allometry in pike reflects variation between lakes in prey availability and relative body depth. Functional Ecology 2001; 15: 754-762. 